\title{
O gênero Vaucheria D.C. (Vaucheriaceae, Xanthophyceae) no Sul do Brasil: aspectos taxonômicos e ecológicos
}

\author{
Aurélio Fajar Tonetto ${ }^{1,3}$, Cleto Kaveski Peres $^{2}$ \& Ciro Cesar Zanini Branco ${ }^{1}$ \\ ${ }^{1}$ Universidade Estadual Paulista - UNESP, FCL/Assis, Av. Dom Antônio, 2100, Parque Universitário, \\ CEP 19806-000, Assis, SP, Brasil \\ ${ }^{2}$ Universidade Federal da Integração Latino-Americana - UNILA, Av. Tancredo Neves, 6731, \\ Parque Tecnológico Itaipu, CEP 85867-970, Foz do Iguaçu, PR, Brasil \\ ${ }^{3}$ Autor para correspondência: Aurélio Fajar Tonetto, e-mail: aureliofajar@hotmail.com
}

TONETTO, A.F., PERES, C.K. \& BRANCO, C.C.Z. The genus Vaucheria D.C. (Vaucheriaceae, Xanthophyceae) in southern Brazil: taxonomic and ecological aspects. Biota Neotrop. 12(2): http://www.biotaneotropica.org. br/v12n2/en/abstract?inventory+bn02012022012

Abstract: (The genus Vaucheria D.C. [Vaucheriaceae, Xanthophyceae] in Southern Brazil: taxonomic and ecology aspects). Taxonomic survey and environmental occurrence of the genus Vaucheria were investigated in 105 streams from Southern Brazil. Abiotic variables were correlated to species occurrence. Three species were identified according to gametangia characteristics: V. geminata (Vauch.) D.C., V. taylorii Blum and V. sessilis (Vauch.) D.C.; the latter is reported for the first time in Brazil. The results of this survey suggest that Vaucheria is influenced by environmental characteristics, occurring mainly, in neutral to slightly acidic $\mathrm{pH}$, low turbidity, slow current velocity and in partly shaded streams. This combination of variables seems to be the most favorable in the Vaucheria population establishment in lotic habitats independently of biome.

Keywords: distribution, taxonomy, Vaucheria, Vaucheriales, Xanthophyceae.

TONETTO, A.F., PERES, C.K. \& BRANCO, C.C.Z. O gênero Vaucheria D.C. (Vaucheriaceae, Xanthophyceae) no Sul do Brasil: aspectos taxonômicos e ecológicos. Biota Neotrop. 12(2): http://www.biotaneotropica.org.br/ v12n2/pt/abstract?inventory+bn02012022012

Resumo: (O gênero Vaucheria D.C. [Vaucheriaceae, Xanthophyceae] no Sul do Brasil: aspectos taxonômicos e ecológicos). O levantamento taxonômico e ocorrência ambiental do gênero Vaucheria foram investigados em 105 riachos do sul do Brasil. As variáveis abióticas foram correlacionadas com a ocorrência das espécies. Três espécies foram identificadas de acordo com as características do gametângio: V. geminata (Vauch.) D.C., V. taylorii Blum e V. sessilis (Vauch.) D.C., este último sendo relatado pela primeira vez no Brasil. Os resultados deste estudo sugerem que Vaucheria é influenciada pelas características ambientais, ocorrendo principalmente em $\mathrm{pH}$ neutro a levemente ácido, baixa turbidez, velocidade da correnteza lenta e em riachos parcialmente sombreados. Esta combinação de variáveis parece ser a mais favorável para o estabelecimento da população de Vaucheria em ambientes lóticos, independentemente do bioma.

Palavras-chave: distribuição, taxonomia, Vaucheria, Vaucheriales, Xanthophyceae. 


\section{Introdução}

O gênero Vaucheria D.C. reúne organismos algais com talo filamentoso simples ou ramificado, jamais divididos em células por septos transversais (Bicudo \& Menezes 2006, Lee 2008). O gênero é o mais amplamente distribuído e com maior número de espécies dentre aqueles da ordem Vaucheriales. Neste contexto, representantes do gênero Vaucheria são encontrados em diversos ambientes desde nascentes, riachos, rios, lagos, e até mesmo habitats marinhos (Rieth 1980, Entwisle 1988). As aproximadamente 70 espécies reconhecidas para o gênero são diferenciadas pela morfologia dos órgãos reprodutores sexuados (anterídeo e oogônio), um sistema estabelecido por Vaucher (1803). Rieth (1980) e Entwisle (1988), por sua vez, propuseram a subdivisão do gênero em várias seções. Tais propostas, especialmente a de Entwisle (1988), foram corroboradas por Andersen \& Bailey (2002) que estudaram a filogenia do grupo através da análise de sequências de DNA (gene $r b c \mathrm{~L}$ e espaçadores $p s b \mathrm{~A}-r b c \mathrm{~L}$ e RUBISCO entre $r b c \mathrm{~L}$ e $r b c \mathrm{~S}$ ).

No Brasil, diversos trabalhos têm registrado a ocorrência de espécies de Vaucheria, porém, grande parte destes registros recentes insere-se no contexto da descrição da diversidade e de aspectos ecológicos da comunidade de macroalgas lóticas (Necchi Junior \& Pascoaloto 1993, Branco \& Necchi Junior 1996a, b, Necchi Junior et al. 1995, 1997, 2000, Krupek et al. 2008, Peres et al. 2008). Em adição, há outros registros mais antigos. Martius (1833), por exemplo, relatou a ocorrência de V. terrestris (Vaucher) D.C. para os estados de São Paulo e Minas Gerais e de V. dichotoma (L.) Mart. para o estado de São Paulo. Zeller (1876), por sua vez, registrou V. sessilis (Vauch.) DC. in Lam. et DC. para o estado do Rio de Janeiro.

O gênero, para ambientes continentais, possui caráter cosmopolita, com registros de ocorrência em praticamente todas as regiões do mundo (Hoppaugh 1930, Christensen 1956, 1969, Blum 1971, 1972, Entwisle 1988, Aboal 1989, Sarma \& Rattan 1990, Branco \& Necchi Junior 1996a, Necchi Junior et al. 1997, 2000, Schagerl \& Kerschbaumer 2008).

No Brasil, existe somente um estudo no estado de São Paulo abordando especificamente aspectos taxonômicos e ecológicos deste grupo (Necchi Junior et al. 2001). Assim, em vista do conhecimento relativamente escasso, o principal objetivo deste estudo foi contribuir sobre os aspectos da taxonomia e da distribuição ecológica das espécies do gênero Vaucheria ocorrentes em ambientes lóticos, com base em um amplo programa amostral desenvolvido nos biomas mais representativos da região Sul do Brasil. Em adição, procurou-se investigar a influência das variáveis abióticas típicas de cada bioma sobre a ocorrência e distribuição das espécies deste gênero.

\section{Material e Métodos}

As amostragens foram realizadas em 105 segmentos de riachos distribuídos na região Sul do Brasil (Figura 1). A ocorrência de espécies do gênero Vaucheria nos ambientes lóticos foi investigada em 10 unidades de conservação nos quatro biomas mais representativos da região Sul do Brasil. (Figura 1, Tabela 1). O estudo foi conduzido no período com menor precipitação pluviométrica (entre abril a novembro) de 2005 a 2008. Os pontos de amostragens foram visitados uma vez aplicando-se a técnica de transeção (Necchi Junior et al. 1995). Espécimes foram fixados em solução de formaldeído $4 \%$ (Johansson 1982). Amostras representativas foram incorporadas ao Herbário SJRP ou UPCB (Thiers 2012).

As análises microscópicas, medições e fotografias foram executadas com o auxilio de um microscópio trinocular Leica DM 1000 composto por uma câmera de vídeo Leica DFC 280, acoplada a um microcomputador com software Leica IM-50, específico para análise de imagem. Vinte medições aleatórias, para cada população, foram feitas para cada característica morfométrica utilizada na análise taxonômica (Necchi Junior et al. 2001). A identificação específica baseou-se na observação de estruturas sexuais amplamente descritas na literatura especializada (Christensen 1969, Blum 1972, Rieth 1980, Entwisle 1988, Sarma \& Rattan 1990). Na ausência de estruturas reprodutivas, não foi possível proceder a identificação específica e, assim, tais populações foram referidas como coletivas (spp). Para cada táxon identificado foram apresentadas as seguintes informações: descrição, ilustrações, distribuição na região Sul e no Brasil, espécimes examinados, características ambientais (média \pm desvio-padrão) e considerações taxonômicas quando necessárias.

As seguintes variáveis ambientais foram medidas em cada ponto de amostragem: profundidade, velocidade da correnteza, temperatura da água, turbidez, condutividade específica, $\mathrm{pH}$, oxigênio dissolvido e nutrientes (nitrogênio total e ortofosfato). Exceto pelos nutrientes, todas as variáveis ambientais foram medidas em campo. A temperatura da água, a turbidez, a condutividade específica, o $\mathrm{pH}$ e o oxigênio dissolvido foram medidos diretamente com um controlador de qualidade da água Horiba U-10, equipado com uma sonda constituída por múltiplos eletrodos, utilizando-se uma amostra de água coletada no ponto médio do segmento do riacho. A velocidade da correnteza foi medida utilizando-se um fluxômetro mecânico General Oceanics 2030R e a profundidade foi mensurada com uma régua centimétrica. $\mathrm{O}$ nível de radiação incidente nos ambientes estudados foi estimado utilizando-se os procedimentos e as classes de sombreamento (aberto, parcialmente sombreado, sombreado e fortemente sombreado) propostas por DeNicola et al. (1992). Por fim, os nutrientes foram quantificados em laboratório, a partir de uma amostra previamente congelada, utilizando-se um espectrofotômetro Spectroquant Nova 60 e reagentes específicos.

\section{Resultados}

Aspectos taxonômicos - Chave artificial de identificação das espécies de Vaucheria para os ambientes lóticos da região Sul do Brasil:

1. Gametângios não dispostos em gametóforos, anterídeos contíguos a 1-2 oogônios V. sessilis

1. Gametângios dispostos em gametóforos com oogônios arranjados lateralmente a um anterídeo

2. Gametóforo portando somente um par de oogônios V. geminata

2. Gametóforo portando 3 oogônios V. taylorii

Vaucheria geminata (Vauch.) DC. in Lam. et DC., Fl. fran., ed. 3, 2: 62,1805 .

Basiônimo: Ectosperma geminata Vaucher Hist. Conf. d'eau douce: 29, pl. 2, Figura 5, 1803. Figuras 2-3.

Sifões ramificados, monóicos com gametóforos em grupos bissexuais, portando 2 oogônios, raramente 1, dispostos lateralmente a um anterídeo, formando densos tufos verdes sobre o substrato, 39,6-114,8 $\mu \mathrm{m}$ diâm.; pedúnculo do gametóforo raramente mais longo do que os oogônios e curvado para o lado, terminando em um anterídeo; anterídeos circinados, tubulares, (49,4-)54-91,5 $\mu \mathrm{m}$ compr. $\times$ 15,0-36,8(-41,8); oogônios reniformes a ovóides, 60,8-97,8 $\mu \mathrm{m}$ compr. $\times 56,6-85,9 \mu \mathrm{m}$ diâm. Oósporos preenchendo o oogônio, 44,6-85,5 $\mu$ m compr. $\times$ 40,7-72,7 $\mu$ m diâm.

Distribuição no Brasil: São Paulo (Branco \& Necchi Junior 1996a, b, Necchi Junior et al. 2001).

Distribuição no Sul do Brasil: pontos de amostragem 34 e 51. BRASIL. PARANÁ: Paranaguá, Parque Nacional Saint-Hilaire/ Lange, rio das Pombas, 25 39' 18' S and 48 35' 42”' W, 03-VII-2005, 

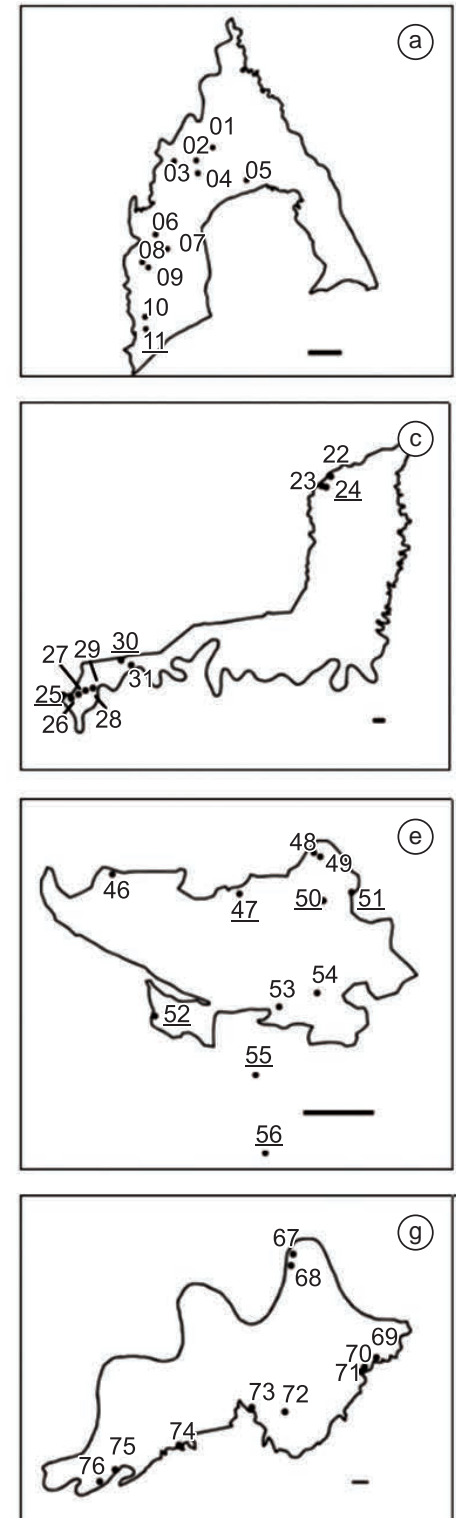
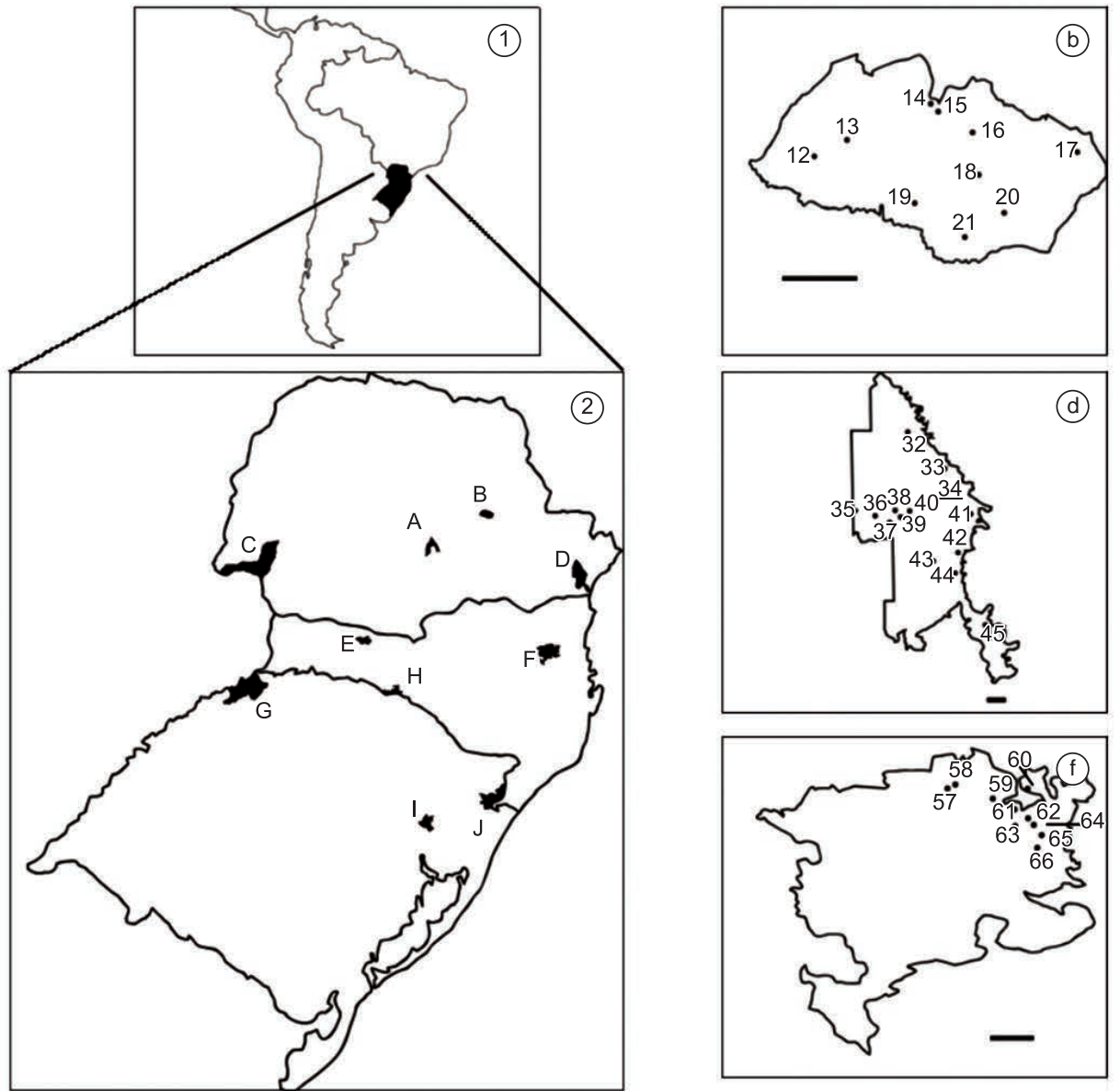

(i)

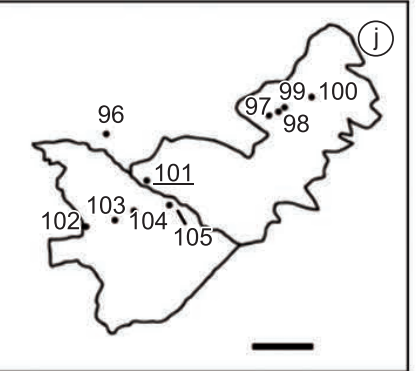

Figura 1. 1.1. Mapa da América do Sul, destacando o Brasil e a região Sul; 1.2 região Sul, destacando os três estados (Paraná, Santa Catarina e Rio Grande do Sul) e a localização das unidades de conservação investigadas; a) Floresta Nacional de Irati; b) Parque Estadual de Vila Velha; c) Parque Nacional do Iguaçú; d) Parque Nacional Saint-Hilaire/Lange; e) Parque Estadual das Araucárias; f) Parque Nacional Serra do Itajaí; g) Parque Florestal Estadual do Turvo; h) Parque Estadual Fritz Plaumann; i) Parque Estadual do Caracol; j) Parque Nacional de Aparados da Serra/ Parque Nacional da Serra Geral. Barras de escalas: $0,5 \mathrm{~km}-\mathrm{a} ; 1 \mathrm{~km}-\mathrm{b}, \mathrm{e}, \mathrm{g}, \mathrm{h}$ e i; $5 \mathrm{~km}$ - c, d e j. Pontos com a presença do gênero Vaucheria D.C. estão sublinhados.

Figure 1. 1.1. Map of South America, especially Brazil and the Southern region, 1.2 South region, highlighting the three states (Paraná, Santa Catarina and Rio Grande do Sul) and location of conservation areas investigated; a) National Forest of Irati; b) State Park of Vila Velha, c) National Park of Iguaçu, d) National Park of Saint-Hilaire/Lange; e) State Park of Araucárias; f) National Park of Serra do Itajaí, g) Turvo State Forest Park; h) State Park of Fritz Plaumann; i) State Park of Caracol, j) National Park of Aparados da Serra / Nacional Park of Serra Geral. Scale bars: 0.5 km - a; 1 km - b, e, g, h and i, 5 km - c, d and J. Points with the presence of genus Vaucheria D.C. are underlined.

C.C.Z. Branco et al. s.n. (UPCB53125); SANTA CATARINA: São Domingos, Parque Estadual das Araucárias, riacho sem denominação, $26^{\circ} 27^{\prime} 21^{\prime \prime} \mathrm{S}$ and $52^{\circ} 33^{\prime} 41^{\prime \prime} \mathrm{W}, 14-\mathrm{VIII}-2007$, C.C.Z. Branco et al. s.n. (SJRP29766).

Características ambientais $(\mathrm{n}=2)$ : temperatura: $19,6 \pm 0,2{ }^{\circ} \mathrm{C}$; condutividade: $38 \pm 1 \mu \mathrm{S} . \mathrm{cm}^{-1}$; velocidade da correnteza: $45 \pm 23 \mathrm{~cm} . \mathrm{s}^{-1}$; turbidez: $8 \pm 8 \mathrm{NTU}$; pH: $6,6 \pm 0,1$; oxigênio dissolvido: $5,4 \pm 0,4 \mathrm{mg} . \mathrm{L}^{-1}$; profundidade: $14 \pm 3 \mathrm{~cm}$; $\mathrm{P}-\mathrm{PO}_{4}$ : $0,07 \pm 0,04 \mathrm{mg} \cdot \mathrm{L}^{-1}$; Ntotal: $1,25 \pm 0,21 \mathrm{mg} \cdot \mathrm{L}^{-1}$.
Vaucheria sessilis (Vauch.) DC. in Lam. et DC., Fl. fran., ed. 3, 2: $63,1805$.

Basiônimo: Ectosperma sessilis Vauch., Hist. Conf. d`eau douce, 31-32, pl. II, Figura 7, 1803.

Sinônimos: Vaucheria bursata (OFM.) C.Ag., fide Entwisle, Phycologia 26: 312, 1987. Conferva bursata (OFM.) C.Ag., Nova Acta Acad. Sci. Imp. Petropol. 3: 96, pl. 2: Figura 10, 1788. Vaucheria ovoidea 
Tonetto, A.F. et al.

Tabela 1. Biomas investigados na região Sul do Brasil com suas respectivas unidades de conservação e número de riachos amostrados.

Table 1. Biomes investigated in southern Brazil with their conservation units and number of streams.

\begin{tabular}{clcc}
\hline Bioma & \multicolumn{1}{c}{ Unidade de conservação } & $\mathbf{N}^{\mathbf{0}}$ de riachos amostrados & Total de riachos por bioma \\
\hline FE & Parque Nacional do Iguaçu, PR & 10 & 29 \\
& Parque Estadual Fritz Plaumann, SC & 9 & 10 \\
& Parque Florestal Estadual do Turvo, RS & 11 & 32 \\
FOM & Floresta Nacional de Irati, PR & 11 \\
& Parque Estadual das Araucárias, SC & 10 \\
FOD & Parque Estadual do Caracol, RS & 14 \\
& Parque Nacional Saint-Hilaire/Lange, PR & 10 & 24 \\
CAM & Parque Nacional da Serra do Itajaí, SC & 10 & 20 \\
& Parque Estadual Vila Velha, PR & 10 & 105 \\
\hline
\end{tabular}

FOM - Floresta Ombrófila Mista, FOD - Floresta Ombrófila Densa, FES - Floresta Estacional Subtropical e CAM - Campos.

FOM - Ombrophilous Mixed Forest, FOD - Ombrophilous Dense Forest, FES - Subtropical Deciduous Forest and CAM - Grasslands.

Hassal, Brit. Freshw. Alg. 1: 57, 1845. Vaucheria repens A.H.Hassal, Ann. Mag. Nat. Hist. 11: 430, 1843. Figuras 4-5.

Sifões ramificados, monóicos com, geralmente, um anterídeo e um oogônio pareados; formando densos tufos verdes sobre o substrato, (37-)57-119,7 $\mu \mathrm{m}$ diâm.; anterídeos circinados, tubulares, 48,0-110,0(-121,4) $\mu \mathrm{m}$ compr. $\times 20,3-30,4 \mu \mathrm{m}$ diâm., fixos ao filamento por um pequeno pedicelo, perpendicular ao filamento; oogônios sésseis, ovóides, (46,3-)66,2-113,2(-122,7) $\mu \mathrm{m}$ compr. $\times$ 59,8-102,4 $\mu$ m diâm. Oósporos preenchendo o oogônio, (42,6-)52,6-112,6 $\mu \mathrm{m}$ compr. $\times(37,6-) 49,2-91,2 \mu \mathrm{m}$ diâm.

Distribuição no Brasil: Rio de Janeiro (Zeller 1876); São Paulo Necchi Junior et al. (2001, como V. bursata).

Distribuição no Sul do Brasil: pontos de amostragem 50, 51, 78 e 92. BRASIL. RIO GRANDE DO SUL: Canela, Parque Estadual do Caracol, riacho da estrada do teleférico, $29^{\circ} 18^{\prime} 33^{\prime \prime} \mathrm{S}$ and 5050’ 48” W, 02-VI-2008, C.C.Z. Branco et al. s.n. (SJRP29726); SANTA CATARINA: Concórdia, Parque Estadual Fritz Plaumann, riacho sem denominação, $27^{\circ} 16^{\prime}$ '57' S and 52 $07^{\circ} 18^{\prime \prime} \mathrm{W}, 20-\mathrm{VIII}-$ 2007, C.C.Z. Branco et al. s.n. (SJRP29722); Idem: São Domingos, Parque Estadual das Araucárias, riacho afluente do rio Jacutinga, $26^{\circ} 27^{\prime} 24$ " S and 52 33' 57' W, 14-VIII-2007, C.C.Z. Branco et al. s.n. (SJRP29765); Idem: riacho sem denominação, 26 27' 21' $\mathrm{S}$ and 52³3' 41”'W, 14-VIII-2007, C.C.Z. Branco et al. s.n. (SJRP29766).

Características ambientais $(n=4)$ : temperatura: $16,1 \pm 3,7{ }^{\circ} \mathrm{C}$; condutividade: $32 \pm 10 \mu \mathrm{S} . \mathrm{cm}^{-1}$; velocidade da correnteza: $48 \pm 33 \mathrm{~cm} \cdot \mathrm{s}^{-1}$; turbidez: $9 \pm 4 \mathrm{NTU}$; pH: $6,7 \pm 0,2$; oxigênio dissolvido: $5,5 \pm 0,9 \mathrm{mg} . \mathrm{L}^{-1}$; profundidade: $12 \pm 3 \mathrm{~cm}$; $\mathrm{P}-\mathrm{PO}_{4}$ : $0,11 \pm 0,05 \mathrm{mg} . \mathrm{L}^{-1}$; Ntotal: $0,68 \pm 0,61 \mathrm{mg} . \mathrm{L}^{-1}$.

Considerações taxonômicas: Vaucheria sessilis já foi registrada no Brasil no trabalho de Necchi Junior et al. (2001). Porém, neste trabalho $V$. sessilis foi citada como $V$. bursata, combinação esta utilizada por Entwisle (1987). A dificuldade em separar essas espécies já foi registrada por Rieth (1963) e Christensen (1969). Porém, Rieth (1980) observou que o nome V. bursata, apesar de mais antigo, era inválido por ser inicialmente dado a um musgo, considerando, assim, este como sinônimo de V. sessilis. Esta mesma interpretação nomenclatural foi utilizada por Johnson \& Merrit (2002) na revisão do grupo nas Ilhas Britânicas, sendo então também adotada no presente estudo.

Vaucheria taylorii Blum, Bull. Torrey Bot. Club 98: 191, Figuras 5-12, 1971. Figuras 6-7.
Sifões ramificados, monóicos, gametóforos em grupos bissexuais, portando comumente 3 oogônios e um anterídeo; formando densos tufos verdes sobre o substrato, (42,9-)64,2-87,3(-115,3) $\mu \mathrm{m}$ diâm. anterídeos curvados, tubulares com 21,1-35,3 $\mu \mathrm{m}$ de diâmetro por 58,0-91,9(-115) $\mu \mathrm{m}$ de comprimento, ultrapassando evidentemente os oogônios; oogônios ovóides a oblongo-ovóides, (40,3-)73,7-86,0 $\mu \mathrm{m}$ compr. $\times(34,2-) 61,0-75,7 \mu \mathrm{m}$ diâm. presos ao gametóforo por pequenos pedúnculos dispostos ao redor do anterídeo. Oósporos preenchendo o oogônio, (43,1-)59,9-75,0 $\mu \mathrm{m}$ compr. $\times(27,9-) 50,8-65,5 \mu$ m diâm.

Distribuição no Brasil: primeira ocorrência para território brasileiro.

Distribuição no Sul do Brasil: ponto de amostragem 82. BRASIL. SANTA CATARINA: Concórdia, Parque Estadual Fritz Plaumann, riacho sem denominação, $27^{\circ} 17^{\prime} 28^{\prime}$ ' S and 52 06' 37' W, 19-VIII2007, C.C.Z. Branco et al. s.n. (SJRP29716).

Características ambientais $(n=1)$ : temperatura: $16,1{ }^{\circ} \mathrm{C}$; condutividade: $33 \mu \mathrm{S} . \mathrm{cm}^{-1}$; velocidade da correnteza: $25 \mathrm{~cm} . \mathrm{s}^{-1}$; turbidez: 2 NTU; pH: 6,7; oxigênio dissolvido: 5,7 mg.L.-1; profundidade: $12 \mathrm{~cm}$; $\mathrm{PO}_{4}$ : 0,1 mg.. $\mathrm{L}^{-1}$; Ntotal: 0,5 mg.L $\mathrm{L}^{-1}$.

Considerações taxonômicas: Vaucheria taylorii é descrita pela primeira vez para o território brasileiro. Apesar de a espécie ocorrer comumente em ambientes terrestres, Johnson \& Merrit (2002) comentaram que esta espécie pode ocorrer, raramente, em ambientes aquáticos, como registrado no presente estudo. A despeito dos poucos registros em ambientes aquáticos, como os caracteres morfológicos concordam perfeitamente com a descrição desta espécie, a identificação foi mantida como V. taylorii.

\section{Vaucheria spp.}

Sifões ramificados, formando densos tufos verdes sobre o substrato, 39,6-114,8 $\mu$ m diâm. Anterídeos e oogônios não observados.

Distribuição no Sul do Brasil: pontos de amostragem 11, 24, 25, 30, 47, 52, 55, 56, 93, 94, 95, 101. BRASIL. RIO GRANDE DO SUL: Canela, Parque Estadual do Caracol, riacho próximo ao castelo sem pregos, $29^{\circ} 16^{\prime} 52^{\prime \prime} \mathrm{S}$ and $50^{\circ} 50^{\prime} 52^{\prime \prime} \mathrm{W}, 03-\mathrm{VI}-$ 2008, C.C.Z. Branco et al. s.n. (SJRP29728); Idem: rio Caracol, $29^{\circ} 18^{\prime} 41^{\prime \prime} \mathrm{S}$ and 50 51' 23" W, 02-VI-2008, C.C.Z. ranco et al. s.n. (SJRP29724); Idem: rio Tiririca, 29 $18^{\prime}$ '59' S and 50 51' 01' W, 02-VI-2008, C.C.Z. Branco et al. s.n. (SJRP29725); Idem: Cambará do Sul, Parque Nacional da Serra Geral, riacho afluente do Perdizes, $29^{\circ} 08^{\prime} 23^{\prime \prime} \mathrm{S}$ and $50^{\circ} 05^{\prime} 21^{\prime}$ ' W, 01-VI-08, C.C.Z. Branco et al. s.n. 


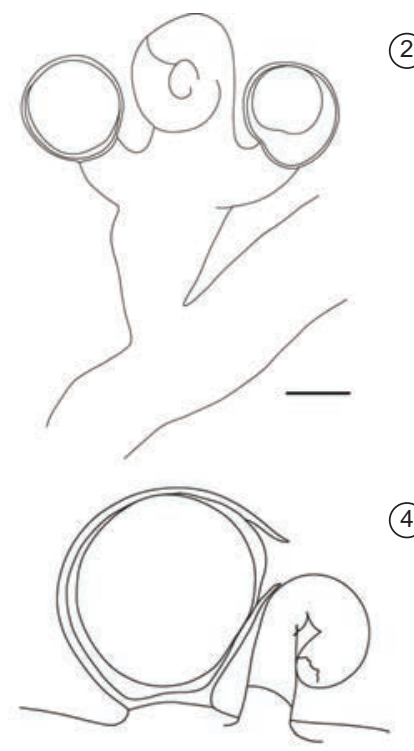

(2)

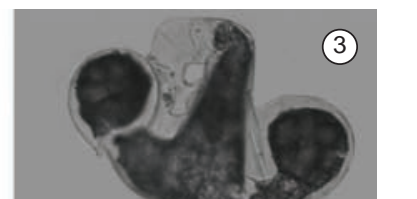

(4)
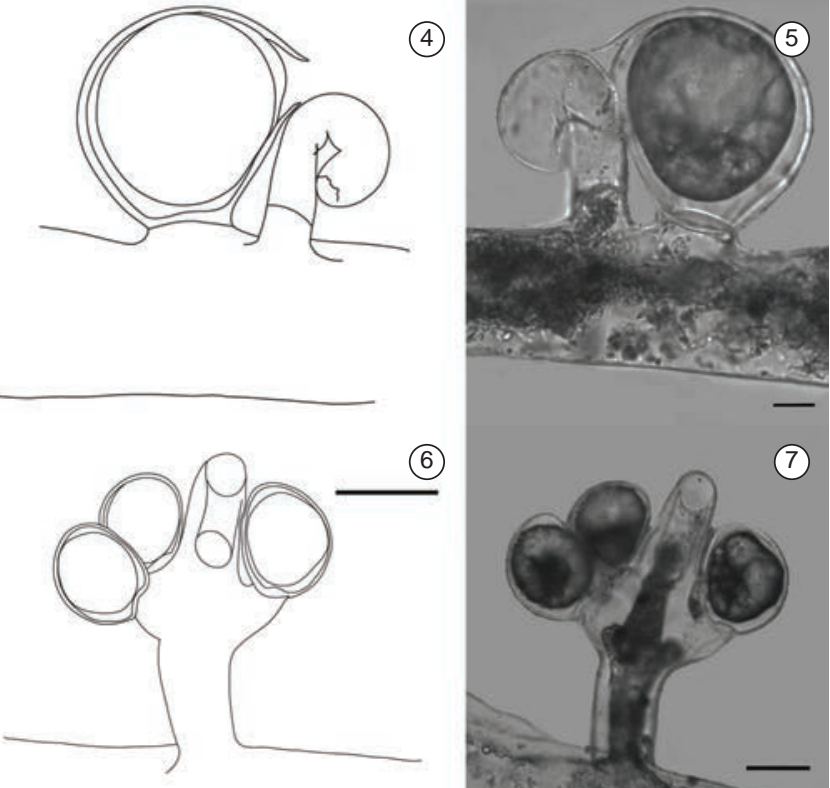

(6)

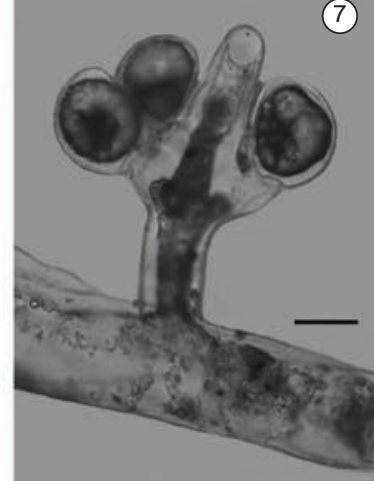

Figura 2-7. Espécies de Vaucheria D.C. encontradas em ambientes lóticos no Sul do Brasil, 2-3. Vaucheria geminata (Vaucher) DC. in Lam. et DC., 4-5. V. sessilis (Vauch.) D.C. in Lam. et D.C., 6-7. V. taylorii Blum. (Barras de escala: $20 \mu \mathrm{m}$ - Figure 5; $50 \mu \mathrm{m}$ - Figure 2-4; 6-7).

Figure 2-7. Species of Vaucheria D.C. found in lotic environments in southern Brazil, 2-3. Vaucheria geminata (Vauch.) D.C. in Lam et DC., 4-5. V. sessilis (Vauch.) D.C. in Lam et DC., 6-7. V. taylorii Blum. (Scale bars: $20 \mu \mathrm{m}$ - Fig 5; $50 \mu \mathrm{m}$ - Figure 2-4, 6-7).

(SJRP29789); SANTA CATARINA: São Domingos, Parque Estadual das Araucárias, riacho na divisa, $26^{\circ} 28^{\prime} 20^{\prime \prime} \mathrm{S}$ and $52^{\circ} 35^{\prime} 26^{\prime \prime} \mathrm{W}$, 15-VIII-2007, C.C.Z. Branco et al. s.n. (SJRP29769); Idem: riacho sem denominação na borda do parque, $26^{\circ} 28^{\prime} 48^{\prime \prime} \mathrm{S}, 52^{\circ} 34^{\prime} 32^{\prime \prime} \mathrm{W}$, 15-VIII-2007, C.C.Z. Branco et al. s.n. (SJRP29770); Idem: riacho sem denominação, 26 29' 25' S and 52 34' 27' 'W, 15-VIII-2007, C.C.Z. Branco et al. s.n. (SJRP29771); Idem: riacho afluente do rio Jacutinga, 26 $27^{\circ}$ '21" S and 52 34' 42" W, 15-VIII-2007, C.C.Z. Branco et al. s.n. (SJRP29772); PARANÁ: Foz do Iguaçu, Parque Nacional do Iguaçu, rio Apepuzinho, 25 32' 01' $\mathrm{S}$ and 54 $4^{\circ} 19^{\prime}$ 36" W, 30-IV-2008, C.C.Z. Branco et al. s.n. (SJRP29753); Idem: rio São João, $25^{\circ} 37^{\prime} 12^{\prime \prime} \mathrm{S}$ and $54^{\circ} 28^{\prime} 34^{\prime}$ ' W, 01-V-2008, C.C.Z. Branco et al. s.n. (SJRP28759); Idem: riacho sem denominação, $25^{\circ} 09^{\prime} 38^{\prime \prime} \mathrm{S}$ and $53^{\circ} 49^{\prime}$ 44” W, 02-V-2008, C.C.Z. Branco et al. s.n. (SJRP29760); Idem: Teixeira Soares, Floresta Nacional de Irati, riacho no talhão $80,25^{\circ} 24^{\prime} 16^{\prime \prime} \mathrm{S}$ and $50^{\circ} 35^{\prime} 28^{\prime \prime} \mathrm{W}, 19-\mathrm{VII}-2005$, C.C.Z. Branco et al. s.n. (SJRP28239).

Características ambientais $(n=12)$ : temperatura: $14,7 \pm 3,6^{\circ} \mathrm{C}$; condutividade: $37 \pm 15 \mu \mathrm{S} . \mathrm{cm}^{-1}$; velocidade da correnteza: $63 \pm 25 \mathrm{~cm} \cdot \mathrm{s}^{-1}$; turbidez: $18 \pm 15 \mathrm{NTU}$; $\mathrm{pH}: 6,3 \pm 0,4$; oxigênio dissolvido: $5,2 \pm 0,8 \mathrm{mg} . \mathrm{L}^{-1}$; profundidade: $15 \pm 7 \mathrm{~cm} ; \mathrm{P}_{-} \mathrm{PO}_{4}$ : $0,10 \pm 0,04 \mathrm{mg} . \mathrm{L}^{-1}$; Ntotal: $1,42 \pm 1,31 \mathrm{mg} . \mathrm{L}^{-1}$.

Distribuição ambiental - A investigação sobre a distribuição do gênero Vaucheria entre os biomas estudados (Tabela 2) revelou ocorrência restrita em apenas aproximadamente $17 \%$ dos riachos. As populações ocorreram, em sua maior parte, nos biomas de floresta ombrófila mista (FOM) (61\%) e floresta estacional (FES) $(31 \%)$. Em cada um desses biomas, $42 \%$ dos riachos em FOM e 31\% dos riachos em FES foram classificados como ambientes parcialmente sombreados, condição em que o gênero foi mais frequente. Biomas tipicamente abertos (Campos, CAM) e sombreados (floresta ombrófila densa, FOD), por sua vez, registraram ocorrência do gênero em apenas um riacho cada.

A maior parte das amostras investigadas (66\%) foi representada por populações estéreis. Dentre as populações com espécimes férteis, Vaucheria sessilis foi a espécie com distribuição mais ampla, sendo encontrada em quatro pontos de amostragens de dois dos biomas investigados. V. geminata, por sua vez, foi encontrada em apenas dois pontos e $V$. taylorii em somente um.

As populações de Vaucheria foram, de modo geral, encontradas nas seguintes condições (média \pm desvio padrão, $\mathrm{n}=18$ ): temperatura $15,6 \pm 3,5{ }^{\circ} \mathrm{C}$, condutividade $36 \pm 12 \mu \mathrm{S} . \mathrm{cm}^{-1}, \mathrm{pH} 6,4 \pm 0,4$, oxigênio dissolvido $5,3 \pm 0,7 \mathrm{mg} . \mathrm{L}^{-1}$, turbidez $14 \pm 13 \mathrm{NTU}$, velocidade da correnteza $56 \pm 27 \mathrm{~cm} \cdot \mathrm{s}^{-1}$, profundidade $14 \pm 6 \mathrm{~cm}$, P-PO $40,10 \pm 0,05$ mg. $\mathrm{L}^{-1}$ e Ntotal 0,98 $\pm 1.06 \mathrm{mg} . \mathrm{L}^{-1}$.

$\mathrm{O}$ gênero apresentou algumas tendências de ocorrência para alguns parâmetros físicos e/ou químicos (Figura 8). Neste contexto, o grupo registrou maior ocorrência em riachos com valores de $\mathrm{pH}$ levemente ácidos a próximo do neutro. Por outro lado, para turbidez a tendência foi contrária à observada para o $\mathrm{pH}$, de modo que o maior número de ocorrências foi registrado em riachos com baixos valores desta variável. Para a velocidade da correnteza, o gênero ocorreu em pontos de amostragem com valores predominantemente baixos. Nenhuma ocorrência foi observada em ambientes classificados como abertos. A maior quantidade de registros de representantes de Vaucheria foi anotada em riachos classificados como parcialmente sombreados, diminuindo gradativamente com o aumento do sombreamento.

\section{Discussão}

O número de espécies identificadas no presente estudo foi o mesmo que o encontrado para o estado de São Paulo (Necchi Junior et al. 2001), ou seja, apenas três espécies. Entretanto, V. taylorii destacase como primeiro registro para território brasileiro, sendo um dos raros registros da espécie em ambientes aquáticos (Johnson \& Merrit 2002). Assim, destaca-se a grande importância da realização de estudos envolvendo o levantamento taxonômico de macroalgas lóticas, de modo a ampliar o conhecimento sobre diversidade deste grupo de organismos, especificamente do gênero Vaucheria de baixa ocorrência. É importante salientar que em ambas as regiões, São Paulo e Sul do Brasil, aproximadamente $60 \%$ das amostras foram representadas por populações estéreis, o que sugere que a reprodução sexual possa não ser o principal meio de crescimento populacional neste grupo de algas em ambientes lóticos. Brawley \& Johnson (1992), de fato, postularam que a ocorrência de fecundação em ambiente natural é rara, o que é coerente com os resultados do presente 
Tonetto, A.F. et al.

Tabela 2. Lista de ocorrência das espécies por bioma/região do sul brasileiro.

Table 2. List of species occurrence by biome/region of southern Brazil.

\begin{tabular}{|c|c|c|c|c|}
\hline Espécies & FOM & FOD & FES & CAM \\
\hline Vaucheria taylorii Blum 1972 & - & - & 1 & - \\
\hline Vaucheria geminata (Vaucher) De Candolle in Lamarck et De Candolle 1805 & 1 & 1 & - & - \\
\hline Vaucheria sessilis (Vaucher) De Candolle in Lamarck et De Candolle 1805 & 3 & - & 1 & - \\
\hline Vaucheria spp. (estéreis) & 8 & - & 3 & 1 \\
\hline Total & 12 & 1 & 5 & 1 \\
\hline
\end{tabular}

C - Campos, FES - Floresta Estacional Subtropical, FOD - Floresta Ombrófila Densa, FOM - Floresta Ombrófila Mista. Os números referem-se ao número de populações (número de pontos de amostragem) registradas em cada bioma.

FOM - Ombrophilous Mixed Forest, FOD - Ombrophilous Dense Forest, FES - Subtropical Deciduous Forest and CAM - Grasslands. The numbers refer to the number of populations (number of sampling points) recorded in each biome.

$\mathrm{pH}$
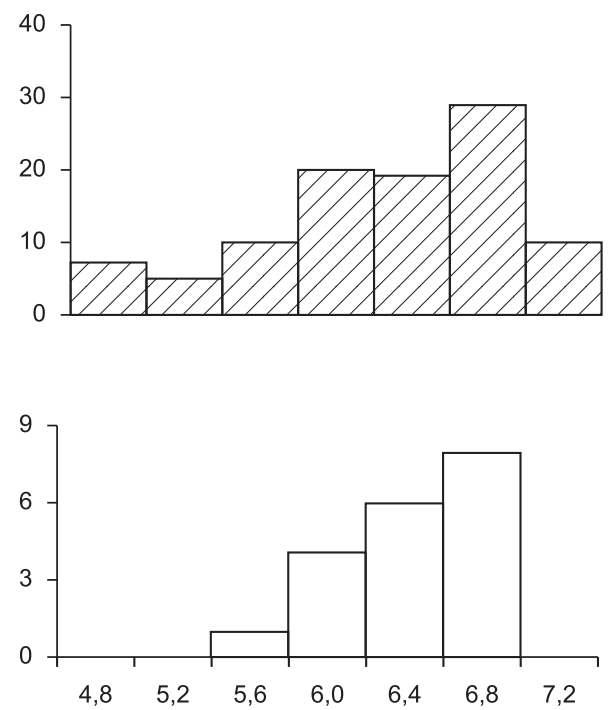

Velocidade correnteza
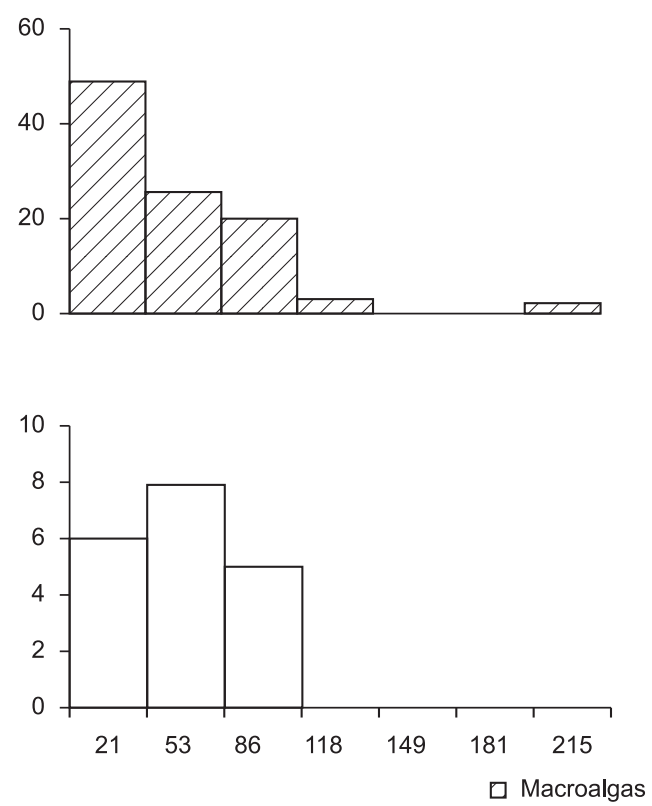

Turbidez
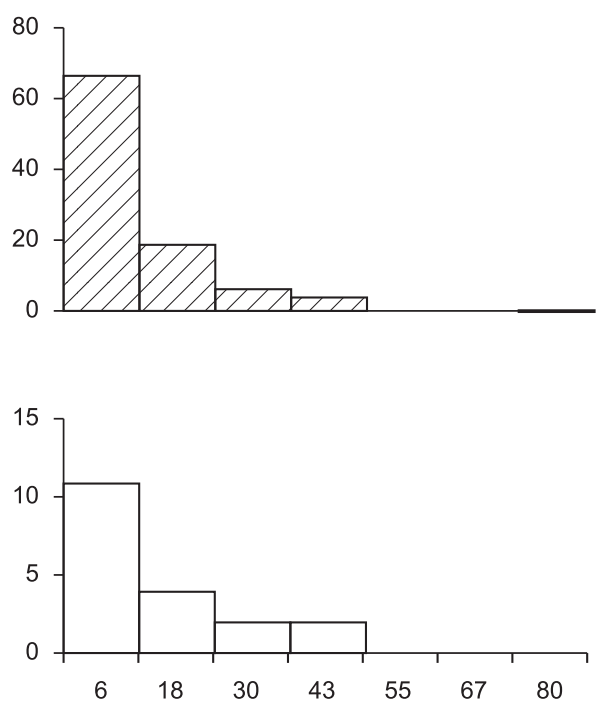

Sombreamento
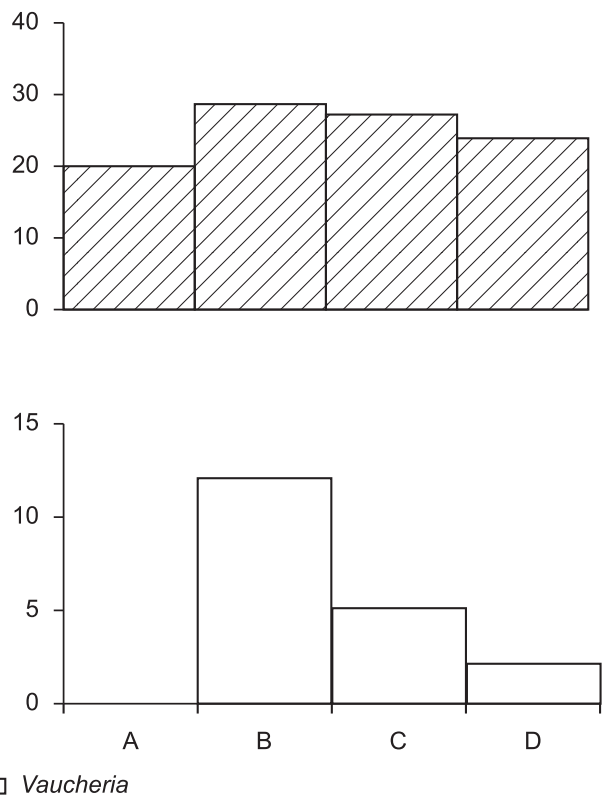

Figura 8. Frequência de distribuição dos riachos amostrados e dos riachos com ocorrência de Vaucheria na região Sul do Brasil de acordo com as variáveis ambientais. (sombreamento: a - aberto, $\mathrm{b}$ - parcialmente sombreado, $\mathrm{c}$ - sombreado e d - fortemente sombreado; Turbidez - NTU; Velocidade da correnteza $-\mathrm{cm} \cdot \mathrm{s}^{-1}$ ). Gráficos pretos - valores referentes a todos os riachos amostrados no Sul do Brasil; Gráficos brancos - valores referentes aos riachos com presença de Vaucheria D.C.

Figure 8. Frequency of distribution of the sampled streams and streams where Vaucheria D.C. occurred in Southern Brazil, according to environmental variables. (Shading: a - open, b - partially shaded, c - shaded and d - heavily shaded; Turbidity - NTU; Current velocity - cm. $\mathrm{s}^{-1}$ ). Black Graphics - values for all sampled streams in southern Brazil, white graphics - values for the streams with the presence of Vaucheria D.C. 
estudo e com registros da literatura (p.ex., Branco \& Necchi Junior 1996a, Necchi Junior et al. 2001, Krupek et al. 2008).

A distribuição ambiental das espécies do gênero em relação ao $\mathrm{pH}$ revelou maior ocorrência nos riachos com valores de $\mathrm{pH}$ próximos do neutro. Assim como os dados de $\mathrm{pH}$, os registros de Vaucheria em ambientes lóticos com baixos valores de turbidez e velocidade da correnteza reforçam informações apresentadas em outros estudos prévios (Branco \& Necchi Junior 1996a, Necchi Junior et al. 2001, Krupek et al. 2008). Assim, de modo geral, os resultados do presente estudo corroboram dados da literatura e sugerem uma tendência de que espécies de Vaucheria ocorrem predominantemente em ambientes lóticos com $\mathrm{pH}$ próximo do neutro e baixos valores de velocidade da correnteza e turbidez.

Com relação aos biomas investigados, a maior ocorrência do grupo notificada para os biomas de FOM e FES está, possivelmente, mais relacionada com a condição de sombreamento dos riachos do que com o bioma propriamente dito. O trabalho de Branco \& Necchi Junior (1996b), por exemplo, conduzido em uma ampla área de FOD da região oriental do estado de São Paulo, registrou a maioria das populações de Vaucheria em riachos classificados como parcialmente sombreados. Necchi Junior et al. (2003, 2008), por outro lado, estudando riachos do bioma de Campos de Altitude da região Sudeste, onde os pontos de amostragem foram predominantemente abertos, não reportaram registros de representantes de Vaucheria. Para o presente estudo, a única ocorrência do gênero em riachos do bioma de Campos de Altitude foi em ponto de amostragem com vegetação marginal exuberante e que proporcionava sombreamento. A ocorrência predominante das populações de Vaucheria em ambientes com sombreamento moderado, observada no presente estudo e na literatura, esta de acordo com a resposta fotossintética observada por Necchi Junior (2004) quando populações do gênero foram expostas experimentalmente a diferentes níveis de irradiância. Neste trabalho, as populações investigadas foram consideradas intermediárias em termos de adaptação à luz (Necchi Júnior 2004). Assim, a disponibilidade de luz no ambiente parece ser um fator relevante para a ocorrência de representantes de Vaucheria, independente do bioma em que se encontram.

\section{Agradecimentos}

Os autores agradecem à CAPES pela bolsa de mestrado de AFT; ao CNPq pelas bolsas de doutorado a CKP (Proc. 141754/2007-9) e de produtividade a CCZB (Proc. 302354/2008-5); a FAPESP pelo financiamento do projeto "Macroalgas do Sul" (Proc. 2007/52608-1) e ao Instituto Chico Mendes e às unidades de conservação mencionadas pela autorização de coleta e apoio logístico durante os trabalhos de campo.

\section{Referências Bibliográficas}

ABOAL, M. 1989. Aportacion al conocimiento de lãs algas epicontinentales Del S.E. de España. V-Xantofíceas (Xanthophyceae P. Allorge et Fritsch, 1935). Bol. Soc. Brot. 62:239-248.

ANDERSEN, R.A. \& BAILEY, J.C. 2002. Phylogenetic analysis of 32 strains of Vaucheria (Xanthophyceae) using the $r b c \mathrm{~L}$ gene and its two flanking spacer regions. J. Phycol. 38:583-592.

BICUDO, C.E.M. \& MENEZES, M. 2006. Gêneros de algas de águas continentais do Brasil chave para identificação e descrição. 2. ed. Rima, São Carlos.

BRANCO, C.C.Z. \& NECCHI JUNIOR, O. 1996a. Survey of stream macroalgae of eastern Atlantic Rainforest of São Paulo State, Southeastern Brazil. Algol. Stud. 80:35-57.
BRANCO, C.C.Z. \& NECCHI JUNIOR, O. 1996b. Distribution of stream macroalgae of eastern Atlantic Rainforest of São Paulo State, Southeastern Brazil. Hydrobiologia 333:139-150.

BRAWLEY, S.H. \& JOHNSON, L.E. 1992. Gametogenesis, Gametes and Zygotes: an Ecological Perspective on Sexual Reproduction in the Algae. Eur. J. Phycol. 27:233-252.

BLUM, J.L. 1971. Notes on American Vaucheriae. Bull. Torrey Bot. Club 98:189-194.

BLUM, J.L. 1972. North American Flora: Vaucheriaceae. The New York Botanical Garden, New York, 51p.

CHRISTENSEN, T. 1956. Studies on the genus Vaucheria III. Bot. Not. 109:275-280.

CHRISTENSEN, T. 1969. Vaucheria collections from Vaucher`s region. Det Kongelige Danske Videnskabernes Selskab Biologiske Skrifter, v.16, p. 25-30.

DENICOLA, D.M., HOGLAND, K.D. \& ROEMER, S.C. 1992. Influence of canopy cover on spectral irradiance and periphyton assemblages in a praire stream. J. N. Am. Benthol. Soc. 11:391-404.

ENTWISLE, T.J. 1987. An evaluation of taxonomic characters in the subsection Sessilis, section Corniculatae, of Vaucheria (Vaucheriaceae, Chrysophyta). Phycologia 26:297-321.

ENTWISLE, T.J. 1988. A monograph of Vaucheria (Vaucheriaceae, Chrysophyta) in south-eastern mainland Australia. Aust. Syst. Bot. 1:1-77.

HOPPAUGH, K. 1930. A taxonomic study of species of the genus Vaucheria collected in California. Am. J. Bot. 17:329-347.

JOHANSSON, C. 1982. Attached algal vegetation in running Waters of Jãmtland, Sweden. Acta Phytogeogr. Suec. 71:1-83.

JOHNSON, L.R. \& MERRIT, R. 2002. Phylum Xanthophyta (Yelow Green Algae). In The Freshwater Algal Flora of the British Isles (D.M. John, B.A. Whitton \& A.J. Brook, eds.). Cambridge University Press, Cambridge, p.245-270.

KRUPEK, R.A., BRANCO, C.C.Z. \& PERES, C.K. 2008. Levantamento florístico das comunidades de macroalgas da bacia do Rio das Pedras, região centro-sul do Estado do Paraná, Sul do Brasil. Hoehnea 35(2):189-208.

LEE, R.E. 2008. Phycology. 4th ed. Cambridge University Press, New York.

MARTIUS, C.F.P. 1833. Algae. In Flora brasiliensis (C.F.P. Martius \& F. Escheweiler, eds.). Sumptibus J.G. Cottae, Stuttgartiae, v.1 Algae, Lichens, Hepaticae, p.1-390.

RIETH, A. 1963. Die algen der chinesisch-deustchen biologischensammelreise durch Nord-und Nordost China 1956. I. Die Vaucheriaceen. 1 Tril. Limnologica 1(4):287-313.

RIETH, A. 1980. Vaucheriales. In Süsswasserflora Von Mittleeuropa, Xanthophyceae II (H. Ettl, J. Gerloff \& H. Heynig, eds.). Gustav Fisher, Stuttgard, p.1-144.

NECCHI JUNIOR, O. 2004. Light-related photosynthetic characteristics of lotic macroalgae. Hydrobiologia 525:139-55.

NECCHI JUNIOR, O., BRANCO, C.C.Z. \& BRANCO, L.H.Z. 1995. Distribution of stream macroalgae in the northwest region of São Paulo State, southeastern Brazil. Hydrobiologia 299:219-230.

NECCHI JUNIOR, O., BRANCO, C.C.Z. \& BRANCO, L.H.Z. 2000. Distribution of stream macroalgae in São Paulo State, southeastern Brazil. Algol. Stud. 97:43-57.

NECCHI JUNIOR, O., BRANCO, L.H.Z. \& BRANCO, C.C.Z. 2003. Ecological distribution of stream macroalgal communities from a drainage basin in the Serra da Canastra National Park, Minas Gerais, southeastern Brazil. Rev. Brasil. Biol. = Braz. J. Biol. 63:635-645.

NECCHI JUNIOR, O., BRANCO, L.H.Z. \& SPEZAMIGLIO D.N. 2008. Distribuição ecológica de comunidades de macroalgas de ambientes lóticos do Parque Nacional de Itatiaia (RJ, MG), Brasil. Rev. Bras. Bot. 31:135-145.

NECCHI JUNIOR, O. \& PASCOALOTO, D. 1993. Seasonal dynamics of macroalgal communities the Preto River Basin, São Paulo, southeastern Brazil. Arch. Hydrobiol. 129:231-252. 
NECCHI JUNIOR, O., PASCOALOTO, D., BRANCO, C.C.Z. \& BRANCO, L.H.Z. 1997. Distribution of stream macroalgae in the northwest region of São Paulo State, southeastern Brazil. Algol. Stud. 84:91-112.

NECCHI JUNIOR, O., SPEZAMIGLIO, D.N.; BRANCO, C.C.Z. \& BRANCO, L.H.Z. 2001. Estudos taxonômicos e distribuição ecológica do gênero Vaucheria (Xanthophyta, Vaucheriaceae) em ecossistemas lóticos do Estado de São Paulo, Brasil. Hoehnea 28(3):231-242.

PERES, C.K., BRANCO, C.C.Z. \& KRUPEK, R.A. 2008. Macroalgas de riachos da Serra da Prata, leste do Paraná, sul do Brasil. Acta Bot. Bras. 22(2):333-344.

SCHAGERL, M. \& KERSCHBAUMER, M. 2008. Autoecology and morphology of selected Vaucheria species (Xanthophyceae). Aquat. Ecol. 43(2):295-303.
SARMA, T.A. \& RATTAN, R.S. 1990. Genus Vaucheria in India. Nova Hedwigia. 51:489-503.

THIERS, B. 2012. Index Herbariorum: A global directory of public herbaria and associated staff. New York Botanical Garden's Virtual Herbarium, New York. http://sweetgum.nybg.org/ih/.

VAUCHER, J.P.E. 1803. Histoire des conferves d'eau douce contenant leurs différens modes de reproduction, et la description de leurs principales espèces, suivie de l'histoire des trémelles et des ulves d'eau douce. J. J. Paschoud, Genéve, p.[i]-xv, [1]-285 [2], pls I-XVII plates.

ZELLER, G. 1876. Algae brasilensis circa Rio de Janeiro a Dr. A. Glaziou. In Symbolae ad floram Brasiliae centralis cognoscendam, 22 (E. Warming, ed.). Videnskabelige Meddelelser frra Dansk naturhistrisk Forening I Kjöbenhavn, p.437-438. 